Kragujevac Journal of Mathematics

Volume 44(3) (2020), Pages 353-368.

\title{
CERTAIN GENERATING MATRIX FUNCTIONS OF LEGENDRE MATRIX POLYNOMIALS USING LIE ALGEBRAIC METHOD
}

\author{
AYMAN SHEHATA ${ }^{1,2}$
}

\begin{abstract}
The main aim of this present paper is to investigate a new of interesting generating matrix relation for Legendre matrix polynomials with the help of a Lie group-theoretic method. Certain properties are well known but some of them are believed to be novel families of matrix differential recurrence relations and generating matrix functions for these matrix polynomials. Special cases of new results are also given here as applications.
\end{abstract}

\section{Introduction, Motivation and Preliminaries}

Special matrix functions are attaining significant results from both the practical and theoretical examples in different fields of Physics, Mathematics and Lie theory. Theories in connection with the unification of generating matrix relations for various special matrix functions are of greater importance in the study of special matrix functions by Lie group theory. The above idea was originally generated by Weisner group-theoretic method and [22-24] also applied this technique to obtain the generating relation. However, the study of special functions from Lie group-theoretic method approach has been obtained generating relations in the books of McBride [12] and Miller [13]. In [17, 18,21], the author has earlier introduced and studied the Legendre matrix polynomials. In $[3,9-11,14-16,19,20]$, certain properties of some special matrix functions via Lie algebra have been proposed as finite series solutions of second-order differential matrix equation.

Motivated by their work, in the present paper, our aim is to establish some results for Legendre matrix polynomials. Here, we give the families of generating matrix

Key words and phrases. Legendre matrix polynomials, Legendre differential matrix equation, generating matrix functions, Lie algebra.

2010 Mathematics Subject Classification. Primary: 33C45, 33C50 Secondary: 33C80, 15A60.

DOI 10.46793/KgJMat2003.353S

Received: March 5, 2018.

Accepted: May 22, 2018. 
functions for Legendre matrix polynomials and the differential recurrence matrix relations for these matrix polynomials are also obtained in section 2 . In section 3 , we study of linear differential operators for Legendre matrix polynomials which generate Lie algebra to apply Weisner's method to obtain some generating matrix relations and apply these linear operators to determine a local representation which makes a one to one correspondence between these Lie algebra with the help of Weisner's method.

Here, the concepts associated with the functional matrix calculus are reviewed. Throughout this article, for a matrix $A \in \mathbb{C}^{N \times N}$, its spectrum $\sigma(A)$ denotes the set of all the eigenvalues of $A$. We denote by $I$ and $\mathbf{O}$ the identity and null matrix in $\mathbb{C}^{N \times N}$, respectively.

Definition $1.1([7])$. For a matrix $A \in \mathbb{C}^{N \times N}$ such that $\sigma(A)$ does not contain 0 or a negative integer $\left(\sigma(A) \cap \mathbb{Z}^{-}=\emptyset\right.$ where $\emptyset$ is an empty set $)$, the matrix form of shifted factorial is defined as

$$
(A)_{n}= \begin{cases}A(A+I) \cdots(A+(n-1) I)=\Gamma(A+n I) \Gamma^{-1}(A), & n \in \mathbb{N} \\ I, & n=0\end{cases}
$$

where $\Gamma(A)$ is an invertible matrix in $\mathbb{C}^{N \times N}$ and $\Gamma^{-1}(A)$ is inverse Gamma matrix function (see [8]).

For $A$ is an arbitrary matrix in $\mathbb{C}^{N \times N}$ and using (1.1), we have the relations (see Defez and Jódar [4])

$$
\begin{aligned}
(A)_{n+k} & =(A)_{n}(A+n I)_{k}=(A)_{k}(A+k I)_{n}, \\
(-n I)_{k} & = \begin{cases}\frac{(-1)^{k} n !}{(n-k) !} I, & 0 \leq k \leq n, \\
\mathbf{0}, & k>n,\end{cases} \\
(A)_{n-k} & = \begin{cases}(-1)^{k}(A)_{n}\left[(I-A-n I)_{k}\right]^{-1}, & 0 \leq k \leq n, \\
\mathbf{0}, & k>n .\end{cases}
\end{aligned}
$$

If $\operatorname{Re}(\mu) \in \sigma(A)$ is not an integer and using (1.1), we have the relation

$$
\Gamma(I-A-n I) \Gamma^{-1}(I-A)=(-1)^{n}\left[(A)_{n}\right]^{-1},
$$

where $\Gamma(I-A)$ is an invertible matrix.

Lemma 1.1. If $A(k, n)$ is a matrix in $\mathbb{C}^{N \times N}$ for $k, n \in \mathbb{N}_{0}$, the relation is satisfied (see Defez and Jódar [4])

$$
\sum_{n=0}^{\infty} \sum_{k=0}^{\infty} A(k, n)=\sum_{n=0}^{\infty} \sum_{k=0}^{n} A(k, n-k) .
$$

Definition 1.2 (Jódar and Cortés [7]). For any matrices $A, B$, and $C$ in $\mathbb{C}^{N \times N}$ such that $C$ is an invertible matrix and for $|z|<1$, the hypergeometric matrix function is defined as follows

$$
\left.{ }_{2} F_{1}(A, B ; C ; z)=\sum_{k=0}^{\infty} \frac{z^{k}}{k !}(A)_{k}(B)\right)_{k}\left[(C)_{k}\right]^{-1}
$$


For $A \in \mathbb{C}^{N \times N}$, we have the relation (see Defez and Jódar [5])

$$
D^{k}\left[t^{A+m I}\right]=(A+I)_{m}(A+I)_{m-k} t^{A+(m-k) I}, \quad k=0,1,2, \ldots
$$

Theorem 1.1. For $|z|<1$ if $A, B$ and $C$ are matrices in $\mathbb{C}^{N \times N}$ where the matrix $C$ satisfies the condition $C+n I$ is an invertible matrix for all integers $n \geq 0$ and $C$, $C-A$ and $C-B$ are positive stable matrices with all matrices are commutative, then the relation

$$
{ }_{2} F_{1}(A, B ; C ; z)=(1-z)^{C-A-B}{ }_{2} F_{1}(C-A, C-B ; C ; z) .
$$

Corollary $1.1([1,2,6])$. Jacobi matrix polynomials have the matrix recurrence relation:

$$
\begin{aligned}
& (x-1)\left[(A+B+n I) \frac{d}{d x} P_{n}^{(A, B)}(x)+(A+n I) \frac{d}{d x} P_{n-1}^{(A, B)}(x)\right] \\
= & (A+B+n I)\left[n P_{n}^{(A, B)}(x)-(A+n I) P_{n-1}^{(A, B)}(x)\right],
\end{aligned}
$$

where $A$ and $B$ are commutative matrices in $\mathbb{C}^{N \times N}$ such that

$\operatorname{Re}(z)>-1, \quad$ for all $z \in \sigma(A)$ and $\operatorname{Re}(w)>-1, \quad$ for all $w \in \sigma(B)$.

Definition 1.3 ([18]). Let $A$ be a matrix in $\mathbb{C}^{N \times N}$ such that

$$
0<\operatorname{Re}(\lambda)<1, \quad \text { for all } \lambda \in \sigma(A) .
$$

Legendre matrix polynomials $P_{n}(x, A)$ is defined by

$$
\begin{aligned}
P_{n}(x, A) & =\sum_{k=0}^{n} \frac{(-1)^{k}(n+k) !}{k !(n-k) !}\left(\frac{1-x}{2}\right)^{k} \Gamma^{-1}(A+k I) \Gamma(A), \quad n \geq 0 \\
& ={ }_{2} F_{1}\left(-n I,(n+1) I ; A ; \frac{1}{2}(1-x)\right), \quad\left|\frac{1-x}{2}\right|<1,
\end{aligned}
$$

such that $A+k I$ is an invertible matrix for all integers $k \geq 0$.

Theorem 1.2 ([18]). For $n \geq 0$, the Legendre matrix polynomials $P_{n}(x, A)$ satisfy the second order differential matrix equation as

$$
\begin{aligned}
& \left(1-x^{2}\right) D^{2} P_{n}(x, A)+2((1-x) I-A) D P_{n}(x, A)+n(n+1) P_{n}(x, A)=\mathbf{0}, \\
& \left|\frac{x-1}{2}\right|<1, \quad D=\frac{d}{d x} .
\end{aligned}
$$

Theorem 1.3 ([18]). For the Legendre matrix polynomials $P_{n}(x, A)$, we have the pure matrix recurrence relation

$$
(A+n I) P_{n+1}(x, A)=(2 n+1) x P_{n}(x, A)+(A-(n+1) I) P_{n-1}(x, A), \quad n \geq 1 .
$$




\section{Some New Results for Legendre Matrix Polynomials}

Here, we derive families of new results for Legendre matrix polynomials with $A$ a matrix in $\mathbb{C}^{N \times N}$ satisfying the condition (1.9). We have the following main results.

Theorem 2.1. The generating matrix functions for the Legendre matrix polynomials are

$$
\sum_{n=0}^{\infty} t^{n} P_{n}(x, A)=(1-t)^{-1}{ }_{2} F_{1}\left(\frac{1}{2} I, I ; A ; \frac{2(x-1) t}{(1-t)^{2}}\right),
$$

for $\left|\frac{2(x-1) t}{(1-t)^{2}}\right|<1,|t|<1$, and

$$
\sum_{n=0}^{\infty} \frac{t^{n}}{n !} P_{n}(x, A)=e^{t}{ }_{1} F_{1}\left((n+1) I ; A ; \frac{(1-x) t}{2}\right), \quad\left|\frac{(1-x) t}{2}\right|<1 .
$$

Proof. From the definition of hypergeometric matrix function and multiplying $(1-t)^{-1}$, we have

$$
\begin{aligned}
& (1-t)^{-1}{ }_{2} F_{1}\left(\frac{1}{2} I, I ; A ; \frac{2(x-1) t}{(1-t)^{2}}\right) \\
= & \sum_{k=0}^{\infty} \frac{2^{k}}{k !}(1-t)^{-(1+2 k) I} t^{k}(I)_{k}\left(\frac{1}{2} I\right)_{k}\left[(A)_{k}\right]^{-1}(x-1)^{k} \\
= & \sum_{n=0}^{\infty} \sum_{k=0}^{\infty} \frac{2^{k} t^{n+k}}{n ! k !}((1+2 k) I)_{n}(I)_{k}\left(\frac{1}{2} I\right)_{k}\left[(A)_{k}\right]^{-1}(x-1)^{k} .
\end{aligned}
$$

From (1.2), we can write that

$$
(I)_{2 k}=2^{2 k}(I)_{k}\left(\frac{1}{2} I\right)_{k}
$$

which implies

$$
\sum_{n=0}^{\infty} \sum_{k=0}^{\infty} \frac{2^{k} t^{n+k}}{n ! k !}((1+2 k) I)_{n} 2^{-2 k}(I)_{2 k}\left[(A)_{k}\right]^{-1}(x-1)^{k} .
$$

Using (1.2), we get

$$
(I)_{n+2 k}=(I)_{2 k}((1+2 k) I)_{n},
$$

which implies

$$
\sum_{n=0}^{\infty} \sum_{k=0}^{\infty} \frac{t^{n+k}}{n ! k !}(I)_{n+2 k}\left[(A)_{k}\right]^{-1}\left(\frac{x-1}{2}\right)^{k} .
$$

Using Lemma 1.1 and replacing $n$ by $n-k$, we find that

$$
\begin{aligned}
& \sum_{n=0}^{\infty} \sum_{k=0}^{n} \frac{t^{n}}{(n-k) ! k !}(I)_{n+k}\left[(A)_{k}\right]^{-1}\left(\frac{x-1}{2}\right)^{k} \\
= & \sum_{n=0}^{\infty} \sum_{k=0}^{n} \frac{n ! t^{n}}{(n-k) ! k !}((n+1) I)_{k}\left[(A)_{k}\right]^{-1}\left(\frac{x-1}{2}\right)^{k} .
\end{aligned}
$$


By using (1.2) in the above equation, we obtain (2.1).

From the definition of hypergeometric matrix function and multiplying $e^{t}$, we have

$$
\begin{aligned}
& e^{t}{ }_{1} F_{1}\left((n+1) I ; A ; \frac{(1-x) t}{2}\right)=e^{t} \sum_{k=0}^{\infty} \frac{1}{k !}((n+1) I)_{k}\left[(A)_{k}\right]^{-1}\left(\frac{(1-x) t}{2}\right)^{k} \\
= & \sum_{n=0}^{\infty} \sum_{k=0}^{\infty} \frac{t^{n+k}}{n ! k !}((n+1) I)_{k}\left[(A)_{k}\right]^{-1}\left(\frac{1-x}{2}\right)^{k} .
\end{aligned}
$$

Using Lemma 1.1 and replacing $n$ by $n-k$ with the help of these Eqs. (1.1), (1.2) and (1.3), we find that

$$
\begin{aligned}
& \sum_{n=0}^{\infty} \sum_{k=0}^{n} \frac{t^{n}}{(n-k) ! k !}((n-k+1) I)_{k}\left[(A)_{k}\right]^{-1}\left(\frac{1-x}{2}\right)^{k} \\
= & \sum_{n=0}^{\infty} \sum_{k=0}^{n} \frac{t^{n}}{(n-k) ! k !}(-1)^{k}((n+1) I)_{k}\left[(A)_{k}\right]^{-1}\left(\frac{1-x}{2}\right)^{k} \\
= & \sum_{n=0}^{\infty} \sum_{k=0}^{n} \frac{1}{n ! k !}((1+n) I)_{k}(-n I)_{k}\left[(A)_{k}\right]^{-1}\left(\frac{1-x}{2}\right)^{k} t^{n}=\sum_{n=0}^{\infty} \frac{t^{n}}{n !} P_{n}(x, A),
\end{aligned}
$$

which completes of the proof (2.2).

Precisely the same manner as described Theorem 2.1 and using (1.2), (1.3) and (1.4), we can prove the following results.

Theorem 2.2. For Legendre matrix polynomials, the following generating matrix functions are

$$
\sum_{n=0}^{\infty} t^{n} P_{n}(x, A)=(1-t)^{-1}{ }_{2} F_{1}\left(-n I, I ; A ; \frac{(1-x)}{2(1-t)}\right)
$$

and

$$
\sum_{n=0}^{\infty} t^{n} P_{n}(x, A)=(1-t)^{-1}{ }_{2} F_{1}\left(I,(n+1) I ; A ; \frac{(x-1) t}{2(1-t)}\right) .
$$

Lemma 2.1. The following equalities for the hypergeometric matrix function satisfy as follows

$$
\begin{aligned}
\frac{d^{n}}{d z^{n}}\left[z^{C-I}{ }_{2} F_{1}(A, B ; C ; z)\right]= & (C-n I)_{n} z^{C-(n+1) I} \\
& \times{ }_{2} F_{1}(A, B ; C-n I ; z),
\end{aligned}
$$

where $C$ and $C-n I$ are invertible matrices.

$$
\begin{aligned}
\frac{d^{n}}{d z^{n}}\left[{ }_{2} F_{1}(A, B ; C ; z)\right]= & (A)_{n}(B)_{n}\left[(C)_{n}\right]^{-1} \\
& \times{ }_{2} F_{1}(A+n I, B+n I ; C+n I ; z),
\end{aligned}
$$


where $C$ and $C+n I$ are invertible matrices, and

$$
\begin{aligned}
\frac{d^{n}}{d z^{n}}\left[(1-z)^{A+B-C}{ }_{2} F_{1}(A, B ; C ; z)\right]= & (C-A)_{n}(C-B)_{n}\left[(C)_{n}\right]^{-1} \\
& \times(1-z)^{A+B-C-n I}{ }_{2} F_{1}(A, B ; C+n I ; z),
\end{aligned}
$$

where $C$ and $C+n I$ are invertible matrices.

Proof. To prove (2.3), from (1.1) and (1.6), we get

$$
\begin{aligned}
\frac{d^{n}}{d z^{n}}\left[z^{C+(k-1) I}\right] & =(C+(k-1) I)(C+(k-2) I) \cdots(C+(k-n) I) z^{C+(k-n+1) I} \\
& =(C)_{k}(C-n I)_{n}\left[(C-n I)_{k}\right]^{-1} z^{C+(k-n-1) I} .
\end{aligned}
$$

Substituting the above expression into the series expression of hypergeometric matrix function, we obtain (2.3).

From (1.5), we get

$$
\begin{aligned}
\frac{d}{d z}{ }_{2} F_{1}(A, B ; C ; z) & =\sum_{k=0}^{\infty} \frac{z^{k}}{k !}(A)_{k+1}(B)_{k+1}\left[(C)_{k+1}\right]^{-1} \\
& =A B^{-1} C^{-1} \sum_{k=0}^{\infty} \frac{z^{k}}{k !}(A+I)_{k}(B+I)_{k}\left[(C+I)_{k}\right]^{-1} \\
& =A B C^{-1}{ }_{2} F_{1}(A+I ; B+I, C+I ; z) .
\end{aligned}
$$

By iteration (2.6), for $n$, one gets (2.4).

In (1.7), we can write

$$
{ }_{2} F_{1}(C-A, C-B ; C ; z)=(1-z)^{A+B-C}{ }_{2} F_{1}(A, B ; C ; z) .
$$

Differentiating with respect to $z$ of $n$ times with the help of this eq. (2.3), we have

$$
\begin{aligned}
& \frac{d^{n}}{d z^{n}}\left[(1-z)^{A+B-C}{ }_{2} F_{1}(A, B ; C ; z)\right] \\
= & \frac{d^{n}}{d z^{n}}\left[{ }_{2} F_{1}(C-A, C-B ; C ; z)\right] \\
= & (C-A)_{n}(C-B)_{n}\left[(C)_{n}\right]_{2}^{-1} F_{1}(C-A+n I, C-B+n I ; C+n I ; z) \\
= & (C-A)_{n}(C-B)_{n}\left[(C)_{n}\right]^{-1}(1-z)^{A+B-C-n I}{ }_{2} F_{1}(A, B ; C+n I ; z),
\end{aligned}
$$

and using (1.7), we have the desired recurrence relation.

Theorem 2.3. The following differential recurrence matrix relations for Legendre matrix polynomials hold true:

$$
\frac{d^{r}}{d x^{r}}\left[(1-x)^{A-I} P_{n}(x, A)\right]=(-1)^{r}(A-r I)_{r}(1-x)^{A-(r+1) I} P_{n}(x, A+r I),
$$

where $A+r I$ is a matrix $\mathbb{C}^{N \times N}$ satisfying the condition (1.9),

$$
\frac{d^{r}}{d x^{r}}\left[P_{n}(x, A)\right]=(-1)^{r} 2^{-r}(-n I)_{r}((n+1) I)_{r}\left[(A)_{r}\right]^{-1}
$$




$$
\times{ }_{2} F_{1}\left((r-n) I,(n+r+1) I: A+r I ; \frac{1-x}{2}\right),
$$

where $A+r I$ is an invertible matrix $\mathbb{C}^{N \times N}$, and

$$
\begin{aligned}
\frac{d^{r}}{d x^{r}}\left[(1+x)^{I-A} P_{n}(x, A)\right]= & (-1)^{r}(A+n I)_{r}(A-(n+1) I)_{r} \\
& \times\left[(A)_{r}\right]^{-1}(1+x)^{I-A-r I} P_{n}(x, A+r I),
\end{aligned}
$$

where $A+r I$ is a matrix $\mathbb{C}^{N \times N}$ satisfying the condition (1.9).

Proof. To prove (2.7), taking $A \rightarrow-n I, B \rightarrow(n+1) I, C \rightarrow A$ and $z \rightarrow \frac{1-x}{2}$ in equation (2.3), we complete the proof.

Taking $z \rightarrow \frac{1-x}{2}, A=-n I, B=(n+1) I$ and $C=A$ in equation (2.4), which completes of the proof (2.8).

Taking $z \rightarrow \frac{1-x}{2}, A \rightarrow-n I, B \rightarrow(n+1) I$ and $C \rightarrow A$ in equation (2.5), theorem can be proved.

Therefore, in (1.8) we interchange $A$ and $B$ and replace $x$ by $-x$ with the help $P_{n}^{(B, A)}(-x)=(-1)^{n} P_{n}^{(A, B)}(x)$ to obtain in the following result.

Corollary 2.1. Jacobi matrix polynomials have the matrix relation:

$$
\begin{aligned}
& (x+1)\left[(A+B+n I) D P_{n}^{(A, B)}(x)-(B+n I) D P_{n-1}^{(A, B)}(x)\right] \\
= & (A+B+n I)\left[n P_{n}^{(A, B)}(x)+(B+n I) P_{n-1}^{(A, B)}(x)\right], \quad n \geq 1, D=\frac{d}{d x} .
\end{aligned}
$$

The relations presented in the following theorem are also interesting.

Theorem 2.4. Legendre matrix polynomials $P_{n}(x, A)$ satisfy the following differential recurrence matrix relations:

$$
\begin{gathered}
(x-1)\left(D P_{n}(x, A)+D P_{n-1}(x, A)\right)=n\left(P_{n}(x, A)-P_{n-1}(x, A)\right), \quad n \geq 1, \\
(x+1)\left((A+(n-1) I) D P_{n}(x, A)+(A-(n+1) I) D P_{n-1}(x, A)\right) \\
=n\left((A+(n-1) I) P_{n}(x, A)-(A-(n+1) I) P_{n-1}(x, A)\right), \quad n \geq 1
\end{gathered}
$$

and

$$
\left(x^{2}-1\right) D P_{n}(x, A)=((1+n x) I-A) P_{n}(x, A)+(A-(n+1) I) P_{n-1}(x, A), \quad n \geq 1 .
$$

Proof. To prove 2.9. In the generating matrix relation (2.1). If we put that

$$
\begin{aligned}
\Phi(x, t, A) & =\sum_{n=0}^{\infty} t^{n} P_{n}(x, A)=(1-t)^{-1}{ }_{2} F_{1}\left(\frac{1}{2} I, I ; A ; \frac{2(x-1) t}{(1-t)^{2}}\right) \\
& =(1-t)^{-1} \Psi(x, t, A),
\end{aligned}
$$


where $\Psi(x, t, A)={ }_{2} F_{1}\left(\frac{1}{2} I, I ; A ; \frac{2(x-1) t}{(1-t)^{2}}\right)$.

Differentiating (2.12) with respect to $x$ and $t$, we obtain

$$
\frac{\partial}{\partial x} \Phi(x, t, A)=2 t(1-t)^{-3} \Psi^{\prime}(x, t, A)
$$

and

$$
\frac{\partial}{\partial t} \Phi(x, t, A)=(1-t)^{-2} \Psi(x, t, A)+2(x-1)(1+t)(1-t)^{-4} \Psi^{\prime}(x, t, A) .
$$

Therefore $\Phi(x, t, A)$ satisfies the partial differential matrix equation

$$
(x-1)(1+t) \frac{\partial}{\partial x} \Phi(x, t, A)-t(1-t) \frac{\partial}{\partial t} \Phi(x, t, A)=-t \Phi(x, t, A) .
$$

Equation (2.13) can be put that

$$
\begin{aligned}
(x-1) \frac{\partial}{\partial x} \Phi(x, t, A)-t \frac{\partial}{\partial t} \Phi(x, t, A)= & -t \Phi(x, t, A)-t^{2} \frac{\partial}{\partial t} \Phi(x, t, A) \\
& -(x-1) t \frac{\partial}{\partial x} \Phi(x, t, A) .
\end{aligned}
$$

Since

$$
\Phi(x, t, A)=\sum_{n=0}^{\infty} t^{n} P_{n}(x, A),
$$

if we differentiate (2.12) with respect to $x$ and $t$, we get

$$
(1-x) \frac{\partial}{\partial x} \Phi(x, t, A)=\sum_{n=0}^{\infty} t^{n}(1-x) \frac{d}{d x} P_{n}(x, A)
$$

and

(2.14) yields that

$$
\frac{\partial}{\partial t} \Phi(x, t, A)=\sum_{n=0}^{\infty} n t^{n-1} P_{n}(x, A)
$$

$$
\begin{aligned}
& \sum_{n=0}^{\infty} t^{n}\left((x-1) \frac{d}{d x} P_{n}(x, A)-n P_{n}(x, A)\right) \\
= & -\sum_{n=0}^{\infty} t^{n+1} P_{n}(x, A)-\sum_{n=0}^{\infty} n t^{n+1} P_{n}(x, A)-\sum_{n=0}^{\infty} t^{n+1}(x-1) \frac{d}{d x} P_{n}(x, A) \\
= & -\sum_{n=1}^{\infty} t^{n} P_{n-1}(x, A)-\sum_{n=0}^{\infty}(n-1) t^{n} P_{n-1}(x, A)-\sum_{n=1}^{\infty} t^{n}(1-x) \frac{d}{d x} P_{n-1}(x, A) \\
= & \sum_{n=0}^{\infty}(-1-n+1) t^{n} P_{n-1}(x, A)-\sum_{n=1}^{\infty} t^{n}(1-x) \frac{d}{d x} P_{n-1}(x, A) \\
= & -\sum_{n=0}^{\infty} n t^{n} P_{n-1}(x, A)-\sum_{n=1}^{\infty} t^{n}(1-x) \frac{d}{d x} P_{n-1}(x, A) .
\end{aligned}
$$

Comparing the coefficients of $t^{n}$, which leads to (2.9). 
If we choose $A=A-I, B=I-A$ in Corollary 2.1, we see that the matrix polynomials $P_{n}^{(A-I, I-A)}(x)$ is $P_{n}^{(A-I, I-A)}(x)=\frac{(A)_{n}}{n !} P_{n}(x, A)$ which leads to the result (2.10).

Let us eliminate $\frac{d}{d x} P_{n-1}(x, A)$ from multiply $(2.9)$ by $(x+1)(A-(n+1) I)$ and multiply $(2.10)$ by $(x-1)$ which gives the result $(2.11)$.

Eliminating $P_{n-1}(x, A)$ from (1.11) and (2.11), one can obtain in the following result.

Theorem 2.5. The differential recurrence matrix relation for Legendre matrix polynomials holds

$$
\begin{aligned}
\left(x^{2}-1\right) D P_{n}(x, A)= & ((1-(n+1) x) I-A) P_{n}(x, A) \\
& -(A+n I) P_{n+1}(x, A) .
\end{aligned}
$$

\section{Group-Theoretic Method for Legendre Matrix Polynomials}

In order to use Weisner's method. Replacing $D$ by $\frac{\partial}{\partial x}, n$ by $y \frac{\partial}{\partial y}$ and $P_{n}(x, A)$ by $P_{n}(x, y, A)=y^{n} P_{n}(x, A)$ in $(1.10)$ is constructed the partial differential matrix equation

$$
\begin{aligned}
& \left(1-x^{2}\right) \frac{\partial^{2}}{\partial x^{2}} P_{n}(x, y, A)+2[(1-x) I-A] \frac{\partial}{\partial x} P_{n}(x, y, A) \\
& +y^{2} \frac{\partial^{2}}{\partial y^{2}} P_{n}(x, y, A)+2 y \frac{\partial}{\partial y} P_{n}(x, y, A)=\mathbf{0} .
\end{aligned}
$$

Thus, $P_{n}(x, y, A)=y^{n} P_{n}(x, A)$ is a solution of the partial differential matrix equation (3.1). Linear differential operators $\mathbb{A}, \mathbb{B}$ and $\mathbb{C}$ are defined as follows

$$
\begin{gathered}
\mathbb{A}=y \frac{\partial}{\partial y} I, \\
\mathbb{B}=\frac{1-x^{2}}{y} \frac{\partial}{\partial x} I+x \frac{\partial}{\partial y} I+\frac{1}{y}(I-A), \quad y \neq 0,
\end{gathered}
$$

and

$$
\mathbb{C}=\left(1-x^{2}\right) y \frac{\partial}{\partial x} I-x y^{2} \frac{\partial}{\partial y} I+((1-x) I-A) y
$$

Then

$$
\begin{aligned}
& \mathbb{A}\left[P_{n}(x, A) y^{n}\right]=n P_{n}(x, A) y^{n}, \\
& \mathbb{B}\left[P_{n}(x, A) y^{n}\right]=-(A-(n+1) I) P_{n-1}(x, A) y^{n-1}
\end{aligned}
$$

and

$$
\mathbb{C}\left[P_{n}(x, A) y^{n}\right]=(A+n I) P_{n+1}(x, A) y^{n+1} .
$$

From (3.2), (3.3) and (3.4), the following theorem can be stated. 
Theorem 3.1. Linear partial differential operators $\mathbb{A}, \mathbb{B}$ and $\mathbb{C}$ as defined in (3.2), (3.3) and (3.4) have the following properties

$$
\text { (i) }[\mathbb{A}, \mathbb{B}]=-\mathbb{B} ; \quad \text { (ii) }[\mathbb{A}, \mathbb{C}]=\mathbb{C} ; \quad \text { (iii) }[\mathbb{B}, \mathbb{C}]=-2 \mathbb{A}-\mathbb{I} \text {, }
$$

where $\mathbb{I}$ is the identity operator, and the notation $[\mathbb{A}, \mathbb{B}]=A B-B A$.

Proof. Now, we proceed to calculate $[\mathbb{A}, \mathbb{B}]$. So that, we consider the action of $\mathbb{A} \mathbb{B}$ on the Legendre matrix polynomials $P_{n}(x, y, A)$

$$
\mathbb{A B} P_{n}(x, y, A)=y \frac{\partial}{\partial y}\left[\frac{I-x^{2}}{y} \frac{\partial}{\partial x} I+x \frac{\partial}{\partial y} I+\frac{1}{y}(I-A)\right] P_{n}(x, y, A) .
$$

Hence, on simplification, we have

$$
\begin{aligned}
\mathbb{A B} P_{n}(x, y, A)= & \left(1-x^{2}\right) \frac{\partial^{2}}{\partial y \partial x} P_{n}(x, y, A)-\frac{1-x^{2}}{y} \frac{\partial}{\partial x} P_{n}(x, y, A) \\
& +x y \frac{\partial^{2}}{\partial y^{2}} P_{n}(x, y, A)+(I-A) \frac{\partial}{\partial y} P_{n}(x, y, A)-\frac{1}{y}(I-A) P_{n}(x, y, A) .
\end{aligned}
$$

In the similar fashion we can operate $\mathbb{B} \mathbb{A}$ on the $P_{n}(x, y, A)$ and simplified as

$$
\begin{aligned}
\mathbb{B} A P_{n}(x, y, A)= & \left(1-x^{2}\right) \frac{\partial^{2}}{\partial x \partial y} P_{n}(x, y, A)+x \frac{\partial}{\partial y} P_{n}(x, y, A) \\
& +x y \frac{\partial^{2}}{\partial y^{2}} P_{n}(x, y, A)+(I-A) \frac{\partial}{\partial y} P_{n}(x, y, A) .
\end{aligned}
$$

Subtracting (3.10) from (3.9) and for $\frac{\partial^{2}}{\partial x \partial y}=\frac{\partial^{2}}{\partial y \partial x}$, we get

$$
\begin{aligned}
{[\mathbb{A}, \mathbb{B}] P_{n}(x, y, A)=} & (\mathbb{A} \mathbb{B}-\mathbb{B} \mathbb{A}) P_{n}(x, y, A)=-\frac{1-x^{2}}{y} \frac{\partial}{\partial x} P_{n}(x, y, A) \\
& -x \frac{\partial}{\partial y} P_{n}(x, y, A)-\frac{1}{y}(I-A) P_{n}(x, y, A) .
\end{aligned}
$$

Further simplifying, we get

$$
[\mathbb{A}, \mathbb{B}] P_{n}(x, y, A)=-\mathbb{B} P_{n}(x, y, A) .
$$

Hence, we have $[\mathbb{A}, \mathbb{B}]=-\mathbb{B}$. Similarly, we can calculate each of the results $[\mathbb{A}, \mathbb{C}]$ and $[\mathbb{B}, \mathbb{C}]$. Thus, the required results are established.

Now, if we operate the partial differential operator $\left(1-x^{2}\right) \mathbb{L}$ on $P_{n}(x, y, A)$, we give

$$
\begin{aligned}
\left(1-x^{2}\right) \mathbb{L} P_{n}(x, y, A)= & \left(1-x^{2}\right)^{2} \frac{\partial^{2}}{\partial x^{2}} P_{n}(x, y, A)+\left(1-x^{2}\right) y^{2} \frac{\partial^{2}}{\partial y^{2}} P_{n}(x, y, A) \\
& +2[(1-x) I-A]\left(1-x^{2}\right) \frac{\partial}{\partial x} P_{n}(x, y, A) \\
& +2 y\left(1-x^{2}\right) \frac{\partial}{\partial y} P_{n}(x, y, A)
\end{aligned}
$$


and

$$
\begin{aligned}
\mathbb{C B} P_{n}(x, y, A)= & \left(1-x^{2}\right)^{2} \frac{\partial^{2}}{\partial x^{2}} P_{n}(x, y, A)+2((1-x) I-A)\left(1-x^{2}\right) \frac{\partial}{\partial x} P_{n}(x, y, A) \\
& +y\left(1-2 x^{2}\right) \frac{\partial}{\partial y} P_{n}(x, y, A)-x^{2} y^{2} \frac{\partial^{2}}{\partial y^{2}} P_{n}(x, y, A) \\
& +(I-A)^{2} P_{n}(x, y, A) .
\end{aligned}
$$

But

$$
\mathbb{A}^{2} P_{n}(x, y, A)=y \frac{\partial}{\partial y}\left(y \frac{\partial}{\partial y}\right) P_{n}(x, y, A)=y^{2} \frac{\partial^{2}}{\partial y^{2}} U_{n}(x, y, A)+y \frac{\partial}{\partial y} P_{n}(x, y, A) .
$$

Hence, we get

$$
\begin{aligned}
\left(1-x^{2}\right) \mathbb{L} P_{n}(x, y, A)-\mathbb{C B} P_{n}(x, y, A)= & y^{2} \frac{\partial^{2}}{\partial y^{2}} P_{n}(x, y, A)+y \frac{\partial}{\partial y} P_{n}(x, y, A) \\
& -(I-A)^{2} P_{n}(x, y, A)
\end{aligned}
$$

which can be express as:

$$
\left(1-x^{2}\right) \mathbb{L} P_{n}(x, y, A)=\left[\mathbb{C B}+\mathbb{A}^{2}-(I-A)^{2} \mathbb{I}\right] P_{n}(x, y, A) .
$$

Since, $P_{n}(x, y, A)$ is the Legendre matrix polynomials, we conclude that

$$
\left(1-x^{2}\right) \mathbb{L}=\mathbb{C B}+\mathbb{A}^{2}-(I-A)^{2} \mathbb{I} .
$$

Now, we show that

$$
\begin{aligned}
& {\left[\left(1-x^{2}\right) \mathbb{L}, \mathbb{A}\right] P_{n}(x, y, A) } \\
= & \left(\left(1-x^{2}\right) \mathbb{L} \mathbb{A}-\mathbb{A}\left(1-x^{2}\right) \mathbb{L}\right) P_{n}(x, y, A) \\
= & \left(\left(\mathbb{C B}+\mathbb{A}^{2}-(I-A)^{2} \mathbb{I}\right) \mathbb{A}-\mathbb{A}\left(\mathbb{C B}+\mathbb{A}^{2}-(I-A)^{2} \mathbb{I}\right)\right) P_{n}(x, y, A) \\
= & (\mathbb{C} \mathbb{B}-\mathbb{A} \mathbb{C B}) P_{n}(x, y, A) .
\end{aligned}
$$

Also, with the aid of (3.8), we have

$$
\mathbb{C B} A-\mathbb{A} \mathbb{C}=\mathbb{C B A}-(\mathbb{C}+\mathbb{C} \mathbb{A}) \mathbb{B}=\mathbb{C B}-\mathbb{C B}=\mathbf{0} .
$$

So that from (3.11), we get

$$
\left[\left(1-x^{2}\right) \mathbb{L}, \mathbb{A}\right]=\mathbf{0} .
$$

Hence, we proved that $\left(1-x^{2}\right) \mathbb{L}$ commute with $\mathbb{A}$. In a similar manner, we can calculate of the operator $\left(1-x^{2}\right) \mathbb{L}$ commute with each of the differential operators $\mathbb{B}$ and $\mathbb{C}$. Thus, we can give in the following.

Theorem 3.2. The operator $\left(1-x^{2}\right) \mathbb{L}$ commute with each of the linear differential operators $\mathbb{A}, \mathbb{B}$ and $\mathbb{C}$ defined in (3.5), (3.6) and (3.7) as follows
(i) $\left[\left(1-x^{2}\right) \mathbb{L}, \mathbb{A}\right]=\mathbf{0}$,
(ii) $\left[\left(1-x^{2}\right) \mathbb{L}, \mathbb{B}\right]=\mathbf{0}$,
(iii) $\left[\left(1-x^{2}\right) \mathbb{L}, \mathbb{C}\right]=\mathbf{0}$. 
The extended forms of the transformation groups generated by the differential operators $\mathbb{A}, \mathbb{B}$ and $\mathbb{C}$ are given by

$$
\begin{aligned}
& e^{a \mathbb{A}} f(x, y, A)=f\left(x, e^{a} y, A\right), \\
& e^{b \mathbb{B}} f(x, y, A)=f\left(\frac{x y+b}{\sqrt{y^{2}+2 b x y+b^{2}}}, \sqrt{y^{2}+2 b x y+b^{2}}, A\right),
\end{aligned}
$$

for $\left|y^{2}+2 b x y\right|<b^{2},\left|\frac{y}{2 b x}\right|<1$ and

$$
e^{c \mathbb{C}} f(x, y, A)=\left(c^{2} y^{2}+2 c x y+1\right)^{-\frac{1}{2}} f\left(\frac{x+c y}{\sqrt{c^{2} y^{2}+2 c x y+1}}, \frac{y}{\sqrt{c^{2} y^{2}+2 c x y+1}}, A\right),
$$

where $\left|c^{2} y^{2}+2 c x y\right|<1$ and $\left|\frac{c y}{2 x}\right|<1, f(x, y, A)$ is an arbitrary matrix function, and $a, b$ and $c$ are arbitrary constants.

From the above equations, we get

$$
\begin{aligned}
e^{c \mathbb{C}} e^{b \mathbb{B}} e^{a \mathbb{A}} f(x, y, A)= & f\left(\frac{y(x+c y)+b\left(c^{2} y^{2}+2 c x y+1\right)}{\sqrt{c^{2} y^{2}+2 c x y+1} \sqrt{b^{2}\left(c^{2} y^{2}+2 c x y+1\right)+2 b y(x+c y)+y^{2}}},\right. \\
& \left.e^{a} \frac{\sqrt{b^{2}\left(c^{2} y^{2}+2 c x y+1\right)+2 b y(x+c y)+y^{2}}}{\left(c^{2} y^{2}+2 c x y+1\right)^{\frac{3}{2}}}, A\right) .
\end{aligned}
$$

3.1. Generating matrix functions. From $(3.5), P_{n}(x, y, A)=P_{n}(x, A) y^{n}$ is a solution of the system

$$
\mathbb{L} P_{n}(x, y, A)=\mathbf{0} \quad \text { and } \quad(\mathbb{A}-n \mathbb{I}) P_{n}(x, y, A)=\mathbf{0} .
$$

From (3.12), we get

$$
e^{c \mathbb{C}} e^{b \mathbb{B}} e^{a \mathbb{A}}\left(1-x^{2}\right) \mathbb{L}\left[P_{n}(x, A) y^{n}\right]=\left(1-x^{2}\right) \mathbb{L} e^{c \mathbb{C}} e^{b \mathbb{B}} e^{a \mathbb{A}}\left[P_{n}(x, A) y^{n}\right] .
$$

Therefore, the transform $e^{c \mathbb{C}} e^{b \mathbb{B}} e^{a \mathbb{A}}\left[P_{n}(x, A) y^{n}\right]$ is annulled by $\left(1-x^{2}\right) \mathbb{L}$.

If we choose $a=0$ and $P_{n}(x, y, A)=P_{n}(x, A) y^{n}$ in (3.12), we get

$$
\begin{aligned}
& e^{c \mathbb{C}} e^{b \mathbb{B}}\left[P_{n}(x, A) y^{n}\right] \\
= & \left(b^{2}\left(c^{2} y^{2}+2 c x y+1\right)+2 b y(x-c y)+y^{2}\right)^{\frac{1}{2} n}\left(c^{2} y^{2}+2 c x y+1\right)^{-\left(\frac{1}{2}+\frac{3}{2} n\right)} \\
& \times P_{n}\left(\frac{y(x+c y)+b\left(c^{2} y^{2}+2 c x y+1\right)}{\sqrt{c^{2} y^{2}+2 c x y+1} \sqrt{b^{2}\left(c^{2} y^{2}+2 c x y+1\right)+2 b y(x+c y)+y^{2}}}, A\right) .
\end{aligned}
$$

On the other hand we get

$$
\begin{aligned}
e^{c \mathbb{C}} e^{b \mathbb{B}}\left[P_{n}(x, A) y^{n}\right]= & \sum_{m=0}^{\infty} \frac{c^{m}}{m !} \sum_{k=0}^{\infty} \frac{b^{k}}{k !}(A+(n-k) I)_{m}((n+1) I-A)_{k} \\
& \times y^{n-k+m} P_{n-k+m}(x, A) .
\end{aligned}
$$

Equating the results (3.6) and (3.7), we get

$$
\left(b^{2}\left(c^{2} y^{2}+2 c x y+1\right)+2 b y(x+c y)+y^{2}\right)^{\frac{1}{2} n}\left(c^{2} y^{2}+2 c x y+1\right)^{-\left(\frac{1}{2}+\frac{3}{2} n I\right)}
$$




$$
\begin{aligned}
& \times P_{n}\left(\frac{y(x+c y)+b\left(c^{2} y^{2}+2 c x y+1\right)}{\sqrt{c^{2} y^{2}+2 c x y+1} \sqrt{b^{2}\left(c^{2} y^{2}+2 c x y+1\right)+2 b y(x+c y)+y^{2}}}, A\right) \\
= & \sum_{m=0}^{\infty} \sum_{k=0}^{n} \frac{c^{m} b^{k}}{m ! k !}(A+(n-k) I)_{m}((n+1) I-A)_{k} y^{n-k+m} P_{n-k+m}(x, A) .
\end{aligned}
$$

Here, we derive of some interesting results as the particular case of generating matrix relations (2.11), we need to consider three cases.

Case 1: $b=-1, c=0$.

If we substitute $b=-1$ and $c=0$ in (3.13), then it will gives us

$$
e^{-\mathbb{B}} f(x, y, A)=f\left(\frac{x y-1}{\sqrt{y^{2}-2 x y+1}}, \sqrt{y^{2}-2 x y+1}, A\right) .
$$

Hence, if we take $f(x, y, A)=P(x, y, A)=P_{n}(x, A) y^{n}$, we find

$$
-\mathbb{B}\left[P_{n}(x, A) y^{n}\right]=\left(1-2 x y+y^{2}\right)^{\frac{1}{2} n} P_{n}\left(\frac{x y-1}{\sqrt{1-2 x y+y^{2}}}, A\right),
$$

since

$$
\begin{aligned}
\mathbb{B}\left[P_{n}(x, A) y^{n}\right] & =\frac{1-x^{2}}{y} \frac{\partial}{\partial x}\left(P_{n}(x, A) y^{n}\right)+x \frac{\partial}{\partial y}\left(P_{n}(x, A) y^{n}\right) \\
& =((n+1) I-A) P_{n-1}(x, A) y^{n-1} .
\end{aligned}
$$

On another hand, we can expand left-hand side of (3.14) in a series form and then repeated application of (3.6) on the same side of (3.14), we have

$$
e^{-\mathbb{B}}\left[P_{n}(x, A) y^{n}\right]=\sum_{k=0}^{n} \frac{1}{k !}(A-(n+1) I)_{k} P_{n-k}(x, A) y^{n-k} .
$$

Equating expressions (3.14) and (3.15), we get

$$
\sum_{k=0}^{n} \frac{1}{k !}(A-(n+1) I)_{k} P_{n-k}(x, A) y^{n-k}=\left(1-2 x y+y^{2}\right)^{\frac{1}{2} n} P_{n}\left(\frac{x y-1}{\sqrt{1-2 x y+y^{2}}}, A\right) .
$$

Replacing $y^{-1}$ by $t$, we obtain of a generating matrix relation

$$
\begin{aligned}
& \sum_{k=0}^{n} \frac{1}{k !}(A-(n+1) I)_{k} P_{n-k}(x, A) t^{k} \\
= & \left(1-2 x t+t^{2}\right)^{\frac{1}{2} n} P_{n}\left(\frac{x-t}{\sqrt{1-2 x t+t^{2}}}, A\right) .
\end{aligned}
$$

Case 2. If we choose $b=0$ and $c=1$ in (3.13) we have

$$
e^{\mathbb{C}} f(x, y, A)=\left(y^{2}+2 x y+1\right)^{-\frac{1}{2}} f\left(\frac{x+y}{\sqrt{y^{2}+2 x y+1}}, \frac{y}{\sqrt{y^{2}+2 x y+1}}, A\right) .
$$

Hence, if we take $f(x, y, A)=P(x, y, A)=P_{n}(x, A) y^{n}$, we find

$$
e^{\mathbb{C}} P(x, y, A)=\left(y^{2}+2 c x y+1\right)^{-\frac{1}{2}} P\left(\frac{x+y}{\sqrt{y^{2}+2 x y+1}}, \frac{y}{\sqrt{y^{2}+2 x y+1}}, A\right)
$$


and

$$
e^{\mathbb{C}} P(x, y, A)=y^{n}\left(y^{2}+2 c x y+1\right)^{-\frac{n+1}{2}} P\left(\frac{x+y}{\sqrt{y^{2}+2 x y+1}}, A\right) .
$$

Since we have

$$
\begin{aligned}
\mathbb{C}\left[P_{n}(x, A) y^{n}\right]= & \left(1-x^{2}\right) y \frac{\partial}{\partial x}\left(P_{n}(x, A) y^{n}\right)-x y^{2} \frac{\partial}{\partial y}\left(P_{n}(x, A) y^{n}\right) \\
& -x y\left(P_{n}(x, A) y^{n}\right)=(A+n I) P_{n+1}(x, A) y^{n+1} .
\end{aligned}
$$

On other hand, we can expand left hand side of (3.16) in a series form and then repeated application of (3.7) on the same side of (3.16), we have

$$
e^{\mathbb{C}}\left[P_{n}(x, A) y^{n}\right]=\sum_{k=0}^{n} \frac{1}{k !}(A+n I)_{k} P_{n+k}(x, A) y^{n+k} .
$$

Equating expressions (3.16) and (3.17) we get

$$
\sum_{k=0}^{n} \frac{1}{k !}(A+n I)_{k} P_{n+k}(x, A) y^{k}=\left(1+2 x y+y^{2}\right)^{-\frac{n+1}{2}} P_{n}\left(\frac{x+y}{\sqrt{1+2 x y+y^{2}}}, A\right),
$$

and replacing $y$ by $-t$, we get of a generating matrix relation

$$
\begin{aligned}
& \sum_{k=0}^{n} \frac{(-1)^{k}}{k !}(A+n I)_{k} P_{n+k}(x, A) t^{k} \\
= & \left(1-2 x t+t^{2}\right)^{-\frac{n+1}{2}} P_{n}\left(\frac{x-t}{\sqrt{1-2 x t+t^{2}}}, A\right) .
\end{aligned}
$$

Case 3: $b=1, c=-1$.

Let us take $b=1$ and $c=-1$, so that (3.17) becomes

$$
\begin{aligned}
e^{-\mathbb{C}} e^{\mathbb{B}}\left[P_{n}(x, A) y^{n}\right] & =\sum_{r, k=0}^{\infty} \frac{(-1)^{r}}{r ! k !} \mathbb{C}^{r} \mathbb{B}^{k}\left[P_{n}(x, A) y^{n}\right] \\
& =\sum_{r, k=0}^{\infty} \frac{(-1)^{r}}{r ! k !}((A+n I))_{r} \mathbb{B}^{k}\left[P_{n+r}(x, A) y^{n+r}\right] \\
& =\sum_{k=0}^{\infty} \sum_{r}^{n} \frac{(-1)^{r}}{r ! k !} \Gamma(A+n I) \Gamma^{-1}(A+(n-r) I) \mathbb{B}^{k}\left[P_{n}(x, A) y^{n}\right]
\end{aligned}
$$

and

$$
\begin{aligned}
e^{-\mathbb{C}} e^{\mathbb{B}}\left[P_{n}(x, A) y^{n}=\right. & \sum_{k=0}^{\infty} \sum_{r}^{n} \frac{(-1)^{r}}{r ! k !} \Gamma(A+n I) \Gamma^{-1}(A+(n-r) I) \\
& \times(A-(n+1) I)_{k} P_{n-k}(x, A) y^{n-k} .
\end{aligned}
$$

Using (3.13) and (3.18), we get

$$
e^{-\mathbb{C}} e^{\mathbb{B}}\left[P_{n}(x, A) y^{n}\right]=\left(y^{2}-2 x y+1\right)^{-\left(\frac{n+1}{2}\right)} P_{n}\left(\frac{1-x y}{\sqrt{y^{2}-2 x y+1}}, A\right)
$$




$$
=\sum_{k=0}^{\infty} \sum_{r}^{n} \frac{(-1)^{r}}{r ! k !} \Gamma(A+n I) \Gamma^{-1}(A+(n-r) I)(A-(n+1) I)_{k} P_{n-k}(x, A) y^{n-k}
$$

and

$$
\begin{aligned}
& \left(y^{2}-2 x y+1\right)^{-\left(\frac{n+1}{2}\right)} P_{n}\left(\frac{1-x y}{\sqrt{y^{2}-2 x y+1}}, A\right) \\
& =\sum_{k=0}^{\infty} \sum_{r}^{n} \frac{(-1)^{r}}{r ! k !} \Gamma(A+n I) \Gamma^{-1}(A+(n-r) I)(A-(n+1) I)_{k} P_{n-k}(x, A) y^{n-k} .
\end{aligned}
$$

Replacing $y^{-1}$ by $t$ in (3.19) we get

$$
\begin{aligned}
& t^{2 n+1}\left(t^{2}-2 x t+1\right)^{-\left(\frac{n+1}{2}\right)} P_{n}\left(\frac{t-x}{\sqrt{t^{2}-2 t x+1}}, A\right) \\
= & \sum_{k=0}^{\infty} \sum_{r}^{n} \frac{(-1)^{r}}{r ! k !} \Gamma(A+n I) \Gamma^{-1}(A+(n-r) I)(A-(n+1) I)_{k} P_{n-k}(x, A) t^{k} .
\end{aligned}
$$

\section{Conclusion}

A novel approach has been obtained in this paper for studying many interesting results of Legendre matrix polynomials viz certain generating matrix relations, matrix recurrence relation, matrix differential recurrence relation and matrix differential equation. Lie algebra method developed in this work can also be used to study some other Legendre matrix polynomials which play as applications and a vital role in Mathematical Physics in the future. However, the merging of these matrix polynomials with a Lie algebraic techniques is also stimulating for further research work.

Acknowledgements. The author would like to express their deep-felt thanks for the anonymous referees very helpful, informative comments and suggestions which improved the original version of this paper.

\section{REFERENCES}

[1] B. C. A. Altm and E. Erkug-Duman, Families of generating functions for the jacobi and related matrix polynomials, Ars Combin. 117 (2014), 257-273.

[2] R. A. A. Altm and E. Erkug-Duman, On a multivariable extension for the extended jacobi polynomials, J. Math. Anal. Appl. 353 (2009), 121-133.

[3] R. Agarwal and S. Jain, Certain properties of some special matrix functions via lie algebra, International Bulletin of Mathematical Research 2 (2015), 9-15.

[4] E. Defez and L. Jódar, Chebyshev matrix polynomails and second order matrix differential equations, Util. Math. 62 (2002), 107-123.

[5] E. Defez and L. Jódar, Chebyshev matrix polynomails and second order matrix differential equations, Util. Math 62 (2002), 107-123.

[6] E. Defez and A. Law, Jacobi matrix differential equation, polynomial solutions, and their properties, Comput. Math. Appl. 48 (2004), 789-803.

[7] L. Jódar and J. Cortés, On the hypergeometric matrix function, J. Comput. Appl. Math. 99 (1998), 205-217. 
[8] L. Jódar and J. Cortés, Some properties of gamma and beta matrix functions, Appl. Math. Lett. 11 (1998), 89-93.

[9] P. L. R. Kameswari and V. S. Bhagavan, Group theoretic origins of certain generating functions of legendre polynomials, International Journal of Chemical Sciences 13 (2015), 1655-1665.

[10] S. Khan and N. Hassan, 2-variable laguerre matrix polynomials and lie-algebraic techniques, J. Phys. A 43 (2010), Article ID 235204, 21 pages.

[11] S. Khan and N. Raza, 2-variable generalized hermite matrix polynomials and lie algebra representation, Rep. Math. Phys. 66 (2010), 159-174.

[12] E. McBride, Obtaining Generating Functions, Springer-Verlag, New York, Heidelberg, Berlin, 1971.

[13] W. J. Miller, Lie Theory and Special Functions, Academic Press, New York, London, 1968.

[14] M. Shahwan and M. Pathan, Origin of certain generating relations of hermite matrix functions from the view point of lie algebra, Integral Transforms Spec. Funct. 17 (2006), 743-747.

[15] M. Shahwan and M. Pathan, Generating relations of Hermite matrix polynomials by Lie algebraic method, Ital. J. Pure Appl. Math. 25 (2009), 187-192.

[16] A. Shehata, Certain generating matrix relations of generalized Bessel matrix polynomials from the view point of lie algebra method, Bull. Iranian Math. Soc. 44 (2018), 1025-1043.

[17] A. Shehata, Connections between Legendre with Hermite and Laguerre matrix polynomials, Gazi University Journal of Science 28 (2015), 221-230.

[18] A. Shehata, A new kind of Legendre matrix polynomials, Gazi University Journal of Science 29 (2016), 535-558.

[19] A. Shehata, Certain generating relations of konhauser matrix polynomials from the view point of lie algebra method, Politehn. Univ. Bucharest Sci. Bull. Ser. A Appl. Math. Phys. 79 (2017), 123-136.

[20] A. Shehata, Lie algebra and Laguerre matrix polynomials of one variable, General Letters in Mathematics 4 (2018), 1-5.

[21] L. Upadhyaya and A. Shehata, On Legendre matrix polynomials and its applications, International Transactions in Mathematical Sciences and Computer 4 (2011), 291-310.

[22] L. Weisner, Group-theoretic origin of certain generating functions, Pac. J. Appl. Math. 5 (1955), 1033-1039.

[23] L. Weisner, Generating functions for Bessel functions, Canad. J. Math. 11 (1959), 148-155.

[24] L. Weisner, Generating functions for Hermite functions, Canad. J. Math. 11 (1959), 141-147.

${ }^{1}$ Department of Mathematics, Faculty of Science,

Assiut University,

Assiut 71516, EGYPT

${ }^{2}$ Department of Mathematics,

College of Science and Arts, Unaizah,

QASSIM UNIVERSITY,

QAssim, Kingdom of SAudi Arabia

Email address: drshehata20060yahoo.com 